\title{
IRON-DEPLETION PREVENTS BIOFILM FORMATION IN PSEUDOMONAS AERUGINOSA THROUGH TWITCHING MOTILITY AND QUORUM SENSING
}

\author{
Yun Cai, Rui Wang*, Mao-Mao An, Bei-Bei Liang
}

Department of Clinical Pharmacology, the PLA General Hospital, Beijing 100853, People’s Republic of China.

Submitted: December 20, 2008; Returned to authors for corrections: May 06, 2009; Approved: July 22, 2009.

\begin{abstract}
Influence of iron-depletion on twitching motility and quorum sensing (QS) system in $P$. aeruginosa was evaluated. The results demonstrated iron-depletion can retard biofilm formation and increase the twitching motility and expression of QS-related genes, suggesting a potential interaction between twitching motility and QS system in P. aeruginosa biofilm formation.
\end{abstract}

Key words: iron; biofilm; twitching motility; quorum sensing

Pseudomonas aeruginosa is one of the major causes of nosocomial infections (8). In addition, $P$. aeruginosa is a leading pathogen among patients with cystic fibrosis, diffuse panbronchiolitis, and chronic obstructive pulmonary disease $(13,22)$. In patients with these underlying diseases, it can cause chronic infections characterized by the formation of biofilms. Biofilms are composed of a group of bacteria attached to the surfaces and are encased in a hydrated polymeric matrix (4). Bacteria that form biofilms can withstand host immune responses and are much more resistant to antibiotics than their counterparts of nonattached, planktonic bacteria (20). Therefore, infections with biofilm-forming bacteria are persistent and difficult to treat with antibiotics.

P. aeruginosa possesses polar filaments called type IV pili that are involved in attachment and surface translocation by twitching motility (15). Twitching motility, mediated by pilus extension and contraction, is required for the formation of biofilms in $P$. aeruginosa $(10,21)$. Quorum-sensing (QS) system in bacteria detects cell density and regulates the expression of many genes at high cell densities. Many studies demonstrated that $P$. aeruginosa strains lacking functional QS system are less virulent than wild-type strains and form flat, undifferentiated biofilms that are less stable than the differentiated biofilms formed by wild-type $P$. aeruginosa. As a consequence, QS has been suggested as a potential target for new preventive and/or therapeutic strategies of $P$. aeruginosa infections (6).

Iron is essential for most pathogens because iron is an indispensable component of many proteins, especially some enzymes in bacteria. Therefore, iron acquisition from environment is important for the growth and metabolism of $P$. aeruginosa (5). Recently, many studies revealed that iron also play an important role in biofilm formation $(1,23)$. In vitro experiments showed both iron-depletion $(<1 \mu \mathrm{M})$ and ironrepletion $(>100 \mu \mathrm{M})$ retarded biofilm formation (14). Furthermore, some reports showed that the level of free iron is increased in airway secretions of cystic fibrosis patients, and this might be one of the possible reasons for the frequent identification of biofilms in the lungs of these patients (3).

To further determine the role of iron in $P$. aeruginosa 
biofilm formation, we compared the ability of biofilm formation between type IV pili mutant, QS mutant and wildtype strains under the iron-depletion condition. We select irondepletion condition because the concentration of free iron in vivo would not exceed $100 \mu \mathrm{M}$ and thus the results are more relevant for control of biofilm infection.

$P$. aeruginosa wild-type strain PAO1, P. aeruginosa IV pili mutant strain PAO- $\Delta$ pilHIJK and $P$. aeruginosa las $R$ and rhlR mutant strain PAO-JP2 were used in this study. Irondepletion condition in the medium was achieved by addition of iron-specific chelator 2, 2-dipyridyl $(500 \mu \mathrm{M})$ (DPD, Sigma, St. Louis, MO).

Static biofilm experiment was performed as described by Favre-Bonté et al. with some modifications (7). Briefly, PAO1, PAO- $\triangle$ pilHIJK and PAO-JP2 were grown in M-H broth for $6 \mathrm{~h}$ with agitation at $37^{\circ} \mathrm{C}$. Silica gel chips were immersed in the bacterial culture medium without agitation to allow bacteria to adhere and form biofilm. The size of all the chips were $1 \mathrm{~cm}^{2}$ and culture medium were replaced every $48 \mathrm{~h}$ for 7 days of continuous cultivation. Silver staining method is that hydrated polymeric matrix of biofilm can be stained as black by $\mathrm{AgNO}_{3}$ and the biofilm formation can be quantified by gray scale comparison (16). The gray scale of the chips was quantified by using Image pro plus (version 4.01, Media Cybernetics) system.

For twitching motility assay we used Petri dishes with LB agar (Sigma). DPD and $\mathrm{FeCl}_{3}$ (Sigma) were added to the molten agar and the plates were dried overnight at room temperature. PAO1, PAO- $\Delta$ pilHIJK and PAO-JP2 were point inoculated at the bottom of the agar plate. After $48 \mathrm{~h}$, the twitching motility distance along the plastic-agar interface (at the bottom of the agar plate) was measured (2).

Total RNA from PAO1 and PAO- $\Delta$ pilHIJK were isolated from bacterial pellet using a commercial kit (Trizol, Invitrogen, Carlsbad, CA) according to the manufacturer's instructions. Reverse transcriptions were performed using $2 \mu \mathrm{l}$ of random hexamer primers, $2 \mu \mathrm{l}$ of $10 \mathrm{mM}$ dNTPmix $1 \mu \mathrm{l}$ of M-MLV (Promega, Madison, WI) and $1 \mu$ of RNA in a total volume of $30 \mu l$. Reverse transcription reaction was stopped after incubation at $37{ }^{\circ} \mathrm{C}$ for $60 \mathrm{~min}$. Real-time PCR analyses were carried out by using the ABI Prism 7900HT SDS 2.2 software (ABI). The sequences of the genes were obtained from the gene bank of the National Library of Medicine and the primers were designed by using Primer Express software (version 2.0, $\mathrm{ABI})$. The sequences of the primers: lasR (F: 5'-CTG CTC GAG CCG GGC TCG GCC TGT TCT-3' R: 5'-CGG GAT CCG GAT GGC GCT CCA CTC CA-3'), rhlR (F: 5'-CAT GCG CGA GCA GGA GTT GCb-3', R: 5'-TAG GGA TCC TAA TCG AAG CCC AGG CGC-3'), 16sRNA (F: 5'-GAT GAC GGT ACC GGA AGA ATA AGC-3', R: 5'-CCA TGT CAA GGG TAG GTA AGG TTT-3'), Real-time PCR contained $2 \mu \mathrm{l}$ of cDNA, $25 \mu \mathrm{l}$ of SYBR Green I (modified DNA polymerase, SYBR Green I, Optimized PCR buffer, $5 \mathrm{mM} \mathrm{MgCl}_{2}$, Dntp MIX including dUTP) and $1 \mu \mathrm{l}$ of forward and reverse primers. Before amplification, PCR mixtures were heated at $50^{\circ} \mathrm{C}$ for 2 min to prevent carryover of PCR products and then to $95^{\circ} \mathrm{C}$ for $10 \mathrm{~min}$ to denature nucleic acids followed by 40 cycles of $95^{\circ} \mathrm{C}$ for $15 \mathrm{~s}$ and $60^{\circ} \mathrm{C}$ for $1 \mathrm{~min}$. The mRNA level of lasR and $r h l R$ genes in each strain was normalized on the basis of the 16sRNA levels.

Pictures of silica gel chips after silver staining were analyzed by the computer image analysis system (Figure 1). Under normal conditions, wild-type and PAO- $\Delta$ pilHIJK formed similar level of biofilm. However, PAO-JP2 strain formed less biofilms. These results indicated that QS is required for the biofilm formation while pili are not required for biofilm formation under normal condition. Under the irondepletion condition, biofilm formation of all the 3 strains decreased significantly and addition of iron increased biofilm formation although it is still lower than that under normal condition. These results demonstrated that iron is required for biofilm formation in $P$. aeruginosa.

Under normal condition, wild-type strain showed highest twitching motility compared with PAO-JP2 and PAO$\Delta$ pilHIJK strains suggesting that pili and QS are involved in twitching motility. Under iron-depletion condition, twitching motility of PAO1 and PAO-JP2 increased significantly and addition of iron decreased the ability of twitching motility. In 
contrast, twitching motility for PAO- $\Delta$ pilHIJK strain did not change significantly under iron-depletion or iron-repletion conditions (data not shown) suggesting that pili are required for the increased twitching motility under iron-depletion condition.

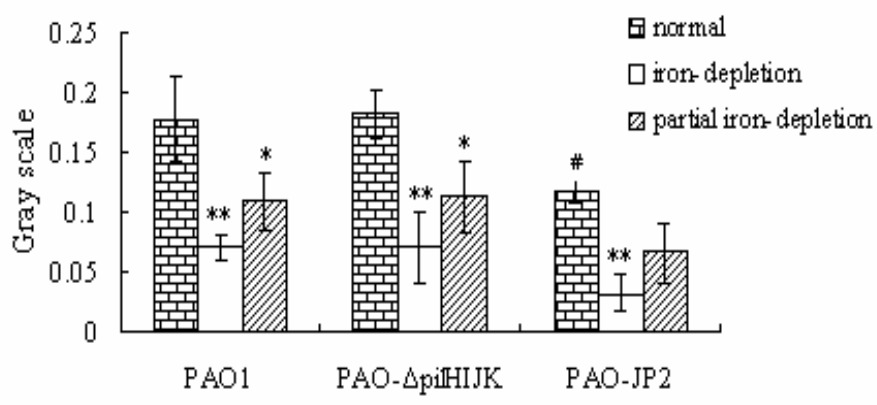

Figure 1. Comparison of biofilm formation under normal (without DPD), iron-depletion (with $500 \mu \mathrm{M}$ DPD) and partial iron-depletion (with $500 \mu \mathrm{M}$ DPD and $25 \mu \mathrm{M} \mathrm{FeCl}_{3}$ ) conditions. The bars represent the means for the 3 tests, and the error bars represent the standard errors of the means. One-factor analysis of variance was conducted. * means $P<0.05$, **means $P<0.01$, both as compared with the same strain in normal condition; \# means $P<0.05$, as compared with PAO1 in normal condition.

Previous studies suggested that twitching motility is necessary for microcolony formation and thus essential for normal biofilm development $(10,15,21)$. However, our study showed that IV pili mutant strain PAO- $\Delta$ pilHIJK formed almost the same amount of biofilm compared to the wild-type strain, PAO1. The most distinct difference is that those studies focused on the initial stage of biofilm development (about 8 24 h), while we studied the mature biofilm formation $(7 \mathrm{~d})$. This may indicate that type IV pilus-mediated twitching motility mediates aggregation of cells in the initial stage of biofilm development, however given enough time ( $7 \mathrm{~d}$ in this study), twitching motility is not required for the formation of mature biofilm. Heydorn et al. (9) also reported that biofilm formed by the PAO- $\Delta$ pilHIJK strain contained a large number of distinct microcolonies that were regularly spaced and almost uniform in size. As the biofilms growing, the microcolonies of the PAO- $\Delta$ pilHIJK strain coalesced, leading to the formation of larger and larger microcolonies. They suggested that microcolonies formed by the PAO- $\Delta$ pilHIJK strain was due to the clonal growth of an individual bacterium in the biofilm because these bacteria were unable to move by twitching motility. Another study also reported that mutant strains defective in twitching motility were not impaired in the early stages of biofilm development and eventually formed mature biofilms, although the morphology were distinguishable from those of wild-type strains (12). Therefore, we hypothesize that another pathway for the formation of microcolonies might exist, and further studies on how PAO- $\Delta$ pilHIJK strain form a mature biofilm are necessary to test this hypothesis.

Biofilm formed by PAO1, PAO- $\Delta$ pilHIJK and PAO-JP2 strains were significantly decreased under iron-depletion condition compared with that under normal condition. We also showed that twitching motility of PAO1 and PAO-JP2 increased under iron-depletion condition. Singh et al. (19) showed that lactoferrin acted as a chelator to bind iron, leading to the twitching and wandering of bacteria across the surface instead of forming cell clusters and biofilms. These findings suggested that iron plays an important role at a critical juncture biofilm development in which bacteria stop roaming as individuals and aggregate into durable communities. Interestingly, as mentioned before, twitching motility is crucial in biofilm formation; however, too much twitching motility prevents biofilm development.

PAO- $\triangle$ pilHIJK strain lacks IV pili and the results showed that twitching motility did not change significantly under irondepletion or iron-repletion conditions. Thus decrease of biofilm formation of PAO- $\Delta$ pilHIJK strain is not due to the decrease in twitching motility. We then determined whether iron-depletion has an effect on QS system, another important system involved in biofilm formation. We showed that upon iron depletion, expression of QS-related genes, lasR and $r h l R$ were significantly increased consistent with the results obtained in other studies $(5,11,23)$. One of the contradictory results is that iron-depletion decreased biofilm formation (Figure 1); 
however, it activated the expression of $l a s R$ and $r h l R$ genes (Figure 2) suggesting an inhibitory role of QS on biofilm development. This could be explained by the results obtained by Patriquin et al. (17) showing that RhlIR/C $\mathrm{C}_{4}-\mathrm{HSL}$ was required for low-Fe-stimulated twitching because rhlIR mutant strain was twitching defective on Fe-limited minimal medium and addition of $\mathrm{C}_{4}$-HSL restores twitching of the rhlI mutant strain. Therefore, under iron-depletion condition, increased rhlIR expression and concomitant $\mathrm{C}_{4}$-HSL production might upregulate a gene(s) leading to the upregulation of twitching and downregulation of biofilm formation.

(a)

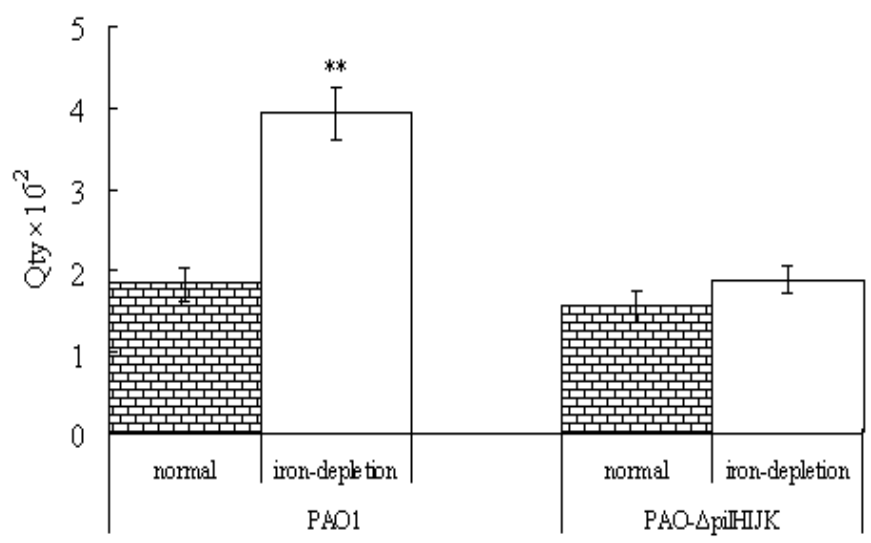

(b)

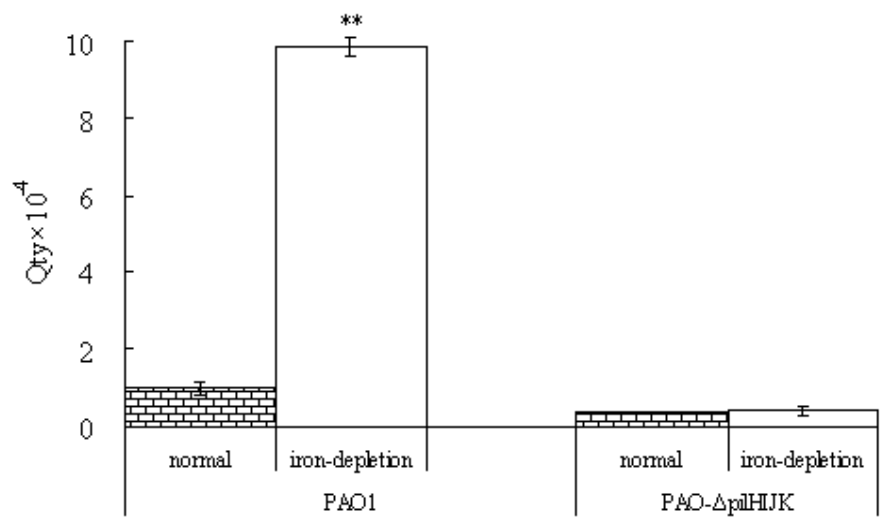

Figure 2. Expression of lasR (a) and $r h l R$ (b) genes in PAO1 and PAO- $\triangle$ pilHIJK stain under normal (without DPD) and irondepletion (with $500 \mu \mathrm{M}$ DPD) conditions. $* *$ means $P<0.01$, as compared with the same strain in normal condition.
Expression of QS-related genes, lasR and rhlR of PAO$\Delta$ pilHIJK does not change significantly under the irondepletion condition. However, in PAO1 strain, iron-depletion significantly enhanced the expression of these two genes (Figure 2). These results indicated that there is correlation between the expression of IV pili and QS-related genes. Pili are encoded by a gene cluster, pilGHIJK that may contain certain gene(s) responsible for the regulation of lasR or rhlR. Reid et al. (18) showed that response of clinical isolates to alterations of environmental iron is quite different to that of PAO1. Thus, we speculate that changes in genome might alter the response of QS to environmental factors.

Based on the results from this study, we concluded that twitching motility is not a prerequisite for the formation of mature biofilm and iron-depletion can decrease the formation of biofilm at least through both twitching motility and QS. The results also indicated there might be a correlation between QS and twitching motility. Further studies on the coordination and regulation of the two key pathways will enhance our understanding of $P$. aeruginosa biofilm formation and identify new targets to control biofilm formation.

\section{REFERENCES}

1. Banin, E.; Brady, K.M.; Greenberg, E.P. (2006). Chelator-induced dispersal and killing of Pseudomonas aeruginosa cells in a biofilm. Appl. Environ. Microbiol. 72(3), 2064-2069.

2. Banin, E.; Vasil, M.L.; Greenberg, E.P. (2005). Iron and Pseudomonas aeruginosa biofilm formation. Proc. Natl. Acad. Sci. U S A. 102(31), 11076-11081.

3. Britigan, B.E.; Hayek, M.B.; Doebbeling, B.N.; Fick, R.B.Jr. (1993). Transferrin and lactoferrin undergo proteolytic cleavage in the Pseudomonas aeruginosa-infected lungs of patients with cystic fibrosis. Infect. Immun. 61(12), 5049-5055.

4. Costerton, J.W.; Stewart, P.S.; Greenberg, E.P. (1999). Bacterial biofilms: a common cause of persistent infections. Science. 284(5418), 1318-1322.

5. Duan, K.; Surette, M.G. (2007). Environmental regulation of Pseudomonas aeruginosa PAO1 Las and Rhl quorum-sensing systems. $J$. Bacteriol 189(13), 4827-36.

6. Favre-Bonté, S.; Chamot, E.; Köhler, T.; Romand, J.A.; van Delden, C. (2007). Autoinducer production and quorum-sensing dependent phenotypes of Pseudomonas aeruginosa vary according to isolation site 
during colonization of intubated patients. BMC Microbiol. 7, 33.

7. Favre-Bonté, S.; Köhler, T.; Van Delden, C. (2003). Biofilm formation by Pseudomonas aeruginosa: role of the C4-HSL cell-to-cell signal and inhibition by azithromycin. J. Antimicrob. Chemother. 52(4), 598-604.

8. Giamarellou, H.; Kanellakopoulou, K. (2008). Current therapies for pseudomonas aeruginosa. Crit. Care. Clin. 24(2), 261-278.

9. Heydorn, A.; Ersbøll, B.; Kato, J.; Hentzer, M.; Parsek, M.R.; TolkerNielsen, T.; Givskov, M.; Molin, S. (2002). Statistical analysis of Pseudomonas aeruginosa biofilm development: impact of mutations in genes involved in twitching motility, cell-to-cell signaling, and stationary-phase sigma factor expression. Appl. Environ. Microbiol. 68(4), 2008-2017.

10. Jenkins, A.T.; Buckling, A.; McGhee, M.; ffrench-Constant, R.H. (2005). Surface plasmon resonance shows that type IV pili are important in surface attachment by Pseudomonas aeruginosa. J. R. Soc. Interface. 2(3), 255-259.

11. Jensen, V.; Löns, D.; Zaoui, C.; Bredenbruch, F.; Meissner, A.; Dieterich, G.; Münch, R.; Häussler, S. (2006). RhlR expression in Pseudomonas aeruginosa is modulated by the Pseudomonas quinolone signal via PhoB-dependent and -independent pathways. J .Bacteriol. 188(24), 8601-8606.

12. Klausen, M.; Heydorn, A.; Ragas, P.; Lambertsen, L.; Aaes-Jørgensen, A.; Molin, S.; Tolker-Nielsen, T. (2003). Biofilm formation by Pseudomonas aeruginosa wild type, flagella and type IV pili mutants. Mol. Microbiol. 48(6), 1511-1524.

13. Murphy, T.F.; Brauer, A.L.; Eschberger, K.; Lobbins, P.; Grove, L.; Cai, X.; Sethi, S. (2008). Pseudomonas aeruginosa in chronic obstructive pulmonary disease. Am. J. Respir. Crit. Care. Med. 177(8), 853-860.

14. Musk, D.J.; Banko, D.A.; Hergenrother, P.J. (2005). Iron salts perturb biofilm formation and disrupt existing biofilms of Pseudomonas aeruginosa. Chem. Biol. 12(7), 789-796.

15. O'Toole, G.A.; Kolter, R. (1998). Flagellar and twitching motility are necessary for Pseudomonas aeruginosa biofilm development. Mol. Microbiol. 30(2), 295-304.

16. Passariello, C.; Berlutti, F.; Selan, L.; Thaller, M.C.; Pezzi, R. (1994). A rapid staining procedure to demonstrate glycocalyx production and bacterial biofilms. New Microbiol. 17(3), 225-230.

17. Patriquin, G.M.; Banin, E.; Gilmour, C.; Tuchman, R.; Greenberg, E.P.; Poole, K. (2008). Influence of quorum sensing and iron on twitching motility and biofilm formation in Pseudomonas aeruginosa. J. Bacteriol. 190(2), 662-671.

18. Reid, D.W.; Kirov, S.M. (2004). Iron, Pseudomonas aeruginosa and cystic fibrosis. Microbiology. 150(Pt 3), 516.

19. Singh, P.K.; Parsek, M.R.; Greenberg, E.P.; Welsh, M.J. (2002). A component of innate immunity prevents bacterial biofilm development. Nature. 417(6888), 552-555.

20. Stewart, P.S.; Costerton, J.W. (2001). Antibiotic resistance of bacteria in biofilms. Lancet. 358(9276), 135-138.

21. Touhami, A.; Jericho, M.H.; Boyd, J.M.; Beveridge, T.J. (2006). Nanoscale characterization and determination of adhesion forces of Pseudomonas aeruginosa pili by using atomic force microscopy. $J$. Bacteriol. 188(2), 370-377.

22. Trisolini, R.; Cancellieri, A.; Paioli, D.; Burzi, M.; Orlandi, P.; Patelli, M. (2008). Sarcoidosis in the setting of idiopathic chronic bronchiolitis with airway colonization from P. aeruginosa: treatment with low-dose macrolides. Intern. Med. 47(6), 537-542.

23. Yang, L.; Barken, K.B.; Skindersoe, M.E.; Christensen, A.B.; Givskov, M.; Tolker-Nielsen, T. (2007). Effects of iron on DNA release and biofilm development by Pseudomonas aeruginosa. Microbiology. 153(Pt 5), 1318-1328. 